\title{
A Youla-Kucera parametrized adaptive feedforward compensator for active vibration control with mechanical coupling ${ }^{\text {th }}$
}

\author{
Ioan Doré Landau ${ }^{\mathrm{a}, *}$, Tudor-Bogdan Airimiţoaie ${ }^{\mathrm{a}, \mathrm{b}}$, Marouane Alma $^{\mathrm{a}}$ \\ ${ }^{a}$ Control system department of Gipsa-lab, St Martin d'Héres, 38402 FRANCE \\ ${ }^{b}$ Faculty of Automatic Control and Computers, University "Politehnica", Bucharest, 060042 ROMANIA
}

\begin{abstract}
Most of the adaptive feedforward vibration (or noise) compensation systems feature an internal "positive feedback" coupling between the compensator system and the correlated disturbance measurement which serves as reference. This may lead to the instability of the system. Instead of the standard IIR structure for the adaptive feedforward compensator, the paper proposes a Youla-Kucera parametrization of the adaptive compensator. The central compensator assures the stability of the system and its performances are enhanced in real time by the direct adaptation of the Youla-Kucera parameters. Theoretical and experimental comparison with recent results obtained using an IIR adaptive feedforward compensators are provided.
\end{abstract}

Keywords: active vibration control, adaptive feedforward compensation, adaptive control, Youla-Kucera parametrization, parameter estimation.

\section{Introduction}

When a correlated measurement with the disturbance is available, adaptive feedforward compensation of broadband vibrations or noise can be considered (Elliott \& Nelson, 1994; Kuo \& Morgan, 1996; Jacobson et al., 2001; Zeng \& de Callafon, 2006). However in many AVC (Active Vibration Control) or ANC (Active Noise Control) systems there is a "positive" feedback coupling between the compensator system and the correlated measurement of the disturbance which serves as reference (Jacobson et al., 2001; Zeng \& de Callafon, 2006; Hu \& Linn, 2000). The positive feedback may destabilize the system. The disturbance is assumed to be unknown and with variable spectral characteristics, but the dynamic models of the AVC and ANC are supposed to be constant and known (these models can be identified).

In Jacobson et al. (2001) and Landau et al. (2011a), algorithms for adapting an IIR feedforward compensator in real time taking into account the presence of the internal positive feedback have been proposed, analyzed and evaluated. In Zeng \& de Callafon (2006), the idea of using a Youla-Kucera parametrization ${ }^{1}$ of the feedforward compensator is illustrated in the context of active noise control. Based on the identification of the system, a stabilizing YK controller is designed. The YK parameters are then updated by using a two time scale indirect procedure: (1) estimation of the Q-filter's parameters over a certain horizon, (2) updating of the controller.

\footnotetext{
The preliminary version of the paper has been accepted at the IFAC World Congress 2011.

${ }^{*}$ Corresponding author. Tel. +33-4-7682-6391. Fax +33-4-7682-6382. Email Ioan-Dore.Landau@ gipsa-lab.grenoble-inp.fr.

${ }^{1}$ Throughout the paper the Youla-Kucera parametrisation will also be called $Q$ (or $Y K$ ) -parametrisation.
}

The main contributions of the present paper with respect to Zeng \& de Callafon (2006) and Landau et al. (2011a) are:

1. the development of a direct real time recursive adaptation algorithm for the Q-parameters of a Youla-Kucera parameterized feedforward filter and the analysis of the stability of the resulting system;

2. possibility to assign the poles of the internal positive closed loop (not possible in Landau et al. (2011a));

3 . easier satisfaction of the positive real condition for stability and convergence;

4. application of the algorithm to an active vibration control system (in Zeng \& de Callafon (2006) an active noise control system is considered) and comparative evaluation with the results given in Landau et al. (2011a).

While the paper is developed in the context of AVC, the results are certainly applicable to ANC systems.

The paper is organized as follows. The system structure is presented in section 2. The algorithm for adaptive feedforward compensation will be developed in section 3 and analysed in section 4. In section 5 the AVC system used for real time experiments is briefly presented. Experimental results obtained on the AVC system are shown in section 6.

\section{Basic equations and notations}

The block diagrams associated with an AVC system are shown in fig. 1 in open loop (1(a)) and when the Youla-Kucera compensator is active (1(b)). For adaptive IIR feedforward compensators see Landau et al. (2011a). $\mathrm{s}(\mathrm{t})$ is the disturbance and $\mathrm{d}(\mathrm{t})$ is the correlated measurement with the disturbance. The primary $(D)$, secondary $(G)$ and reverse (positive coupling) 


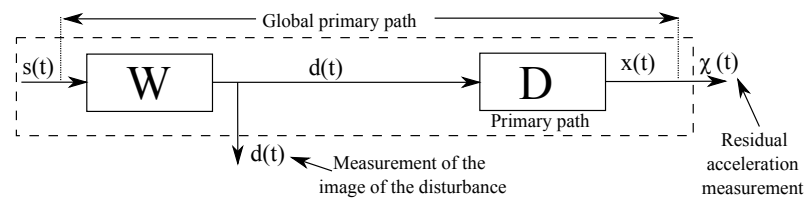

(a)

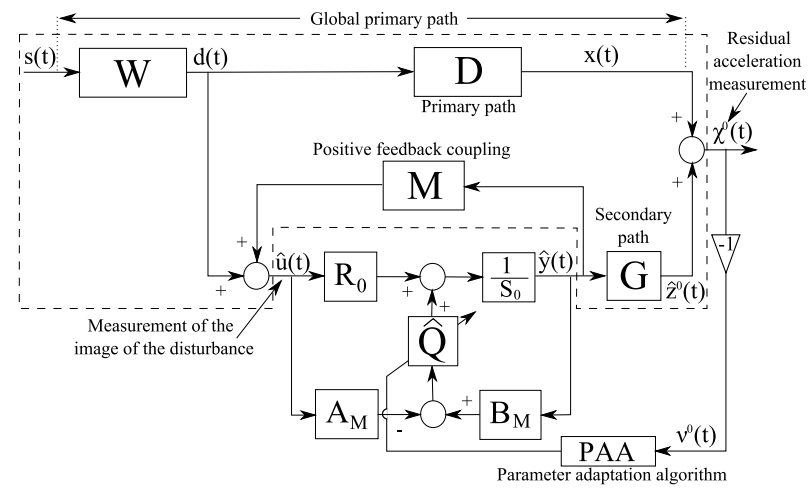

(b)

Figure 1: Feedforward AVC: in open loop (a) and with adaptive feedforward compensator (b)

$(M)$ paths represented in (1(b)) are respectively characterized by the asymptotically stable transfer operators:

$$
\begin{gathered}
D\left(q^{-1}\right)=\frac{B_{D}\left(q^{-1}\right)}{A_{D}\left(q^{-1}\right)}=\frac{b_{1}^{D} q^{-1}+\ldots+b_{n_{B_{D}}}^{D} q^{-n_{B_{D}}}}{1+a_{1}^{D} q^{-1}+\ldots+a_{n_{A_{D}}}^{D} q^{-n_{A_{D}}}} \\
G\left(q^{-1}\right)=\frac{B_{G}\left(q^{-1}\right)}{A_{G}\left(q^{-1}\right)}=\frac{b_{1}^{G} q^{-1}+\ldots+b_{n_{B_{G}}}^{G} q^{-n_{B_{G}}}}{1+a_{1}^{G} q^{-1}+\ldots+a_{n_{A_{G}}}^{G} q^{-n_{A_{G}}}} \\
M\left(q^{-1}\right)=\frac{B_{M}\left(q^{-1}\right)}{A_{M}\left(q^{-1}\right)}=\frac{b_{1}^{M} q^{-1}+\ldots+b_{n_{B_{M}}}^{M} q^{-n_{B_{M}}}}{1+a_{1}^{M} q^{-1}+\ldots+a_{n_{A_{M}}}^{M} q^{-n_{A_{M}}}},
\end{gathered}
$$

with $B_{X}=q^{-1} B_{X}^{*}$ for any $x \in\{D, G, M\} . \hat{G}, \hat{M}$ and $\hat{D}$ denote the identified (estimated) models of G, M and D. The optimal IIR feedforward compensator which will minimize the residual acceleration can be written, using the Youla-Kucera parametrization (Q-parametrization), as

$$
N\left(q^{-1}\right)=\frac{R\left(q^{-1}\right)}{S\left(q^{-1}\right)}=\frac{R_{0}\left(q^{-1}\right)-A_{M}\left(q^{-1}\right) Q\left(q^{-1}\right)}{S_{0}\left(q^{-1}\right)-B_{M}\left(q^{-1}\right) Q\left(q^{-1}\right)}
$$

where the optimal polynomial $Q\left(q^{-1}\right)$ has a FIR structure:

$$
Q\left(q^{-1}\right)=q_{0}+q_{1} q^{-1}+\ldots+q_{n_{Q}} q^{-n_{Q}} .
$$

and $R_{0}\left(q^{-1}\right), S_{0}\left(q^{-1}\right)=1+q^{-1} S_{0}^{*}\left(q^{-1}\right)$ are the polynomials of the central (stabilizing) filter and $A_{M}\left(q^{-1}\right), B_{M}\left(q^{-1}\right)$ are given in (3).

The estimated $Q$ polynomial is denoted ${ }^{2}$ by $\hat{Q}\left(q^{-1}\right)$ or $\hat{Q}\left(\hat{\theta}, q^{-1}\right)$ when it is a linear filter with constant coefficients or $\hat{Q}\left(t, q^{-1}\right)$ during estimation (adaptation).

${ }^{2}$ The complex variable $z^{-1}$ will be used for characterizing the system's behavior in the frequency domain and the delay operator $q^{-1}$ will be used for describing the system's behavior in the time domain.
The input of the feedforward filter (called also reference) is denoted by $\hat{u}(t)$ and it corresponds to the measurement provided by the primary transducer (force or acceleration transducer in AVC or a microphone in ANC). In the absence of the compensation loop (open loop operation) $\hat{u}(t)=d(t)$. The output of the feedforward filter (which is the control signal applied to the secondary path) is denoted by $\hat{y}(t)=\hat{y}(t+1 \mid \hat{\theta}(t+1))$ (a posteriori output). The a priori output $\hat{y}^{0}(t+1)=\hat{y}(t+1 \mid \hat{\theta}(t))$ is given by:

$$
\hat{y}^{0}(t+1)=-S_{0}^{*} \hat{y}(t)+R_{0} \hat{u}(t+1)+\hat{Q}\left(t, q^{-1}\right)\left[B_{M}^{*} \hat{y}(t)-A_{M} \hat{u}(t+1)\right],
$$

where $\hat{y}(t), \hat{y}(t-1), \ldots$ are the "a posteriori" outputs of the feedforward filter generated by

$$
\hat{y}(t+1)=-S_{0}^{*} \hat{y}(t)+R_{0} \hat{u}(t+1)+\hat{Q}\left(t+1, q^{-1}\right)\left[B_{M}^{*} \hat{y}(t)-A_{M} \hat{u}(t+1)\right] .
$$

The measured input to the feedforward filter satisfies the following equation (when feedforward compensation is active)

$$
\hat{u}(t+1)=d(t+1)+\frac{B_{M}^{*}\left(q^{-1}\right)}{A_{M}\left(q^{-1}\right)} \hat{y}(t) .
$$

The unmeasurable value of the output of the primary path is denoted $x(t)$. The unmeasurable "a priori" output of the secondary path will be denoted $\hat{z}^{0}(t+1)$.

$$
\hat{z}^{0}(t+1)=\hat{z}(t+1 \mid \hat{\theta}(t))=\frac{B_{G}^{*}\left(q^{-1}\right)}{A_{G}\left(q^{-1}\right)} \hat{y}(t)
$$

The "a posteriori" unmeasurable value of the output of the secondary path is denoted by:

$$
\hat{z}(t+1)=\hat{z}(t+1 \mid \hat{\theta}(t+1))
$$

The a priori adaptation error is defined as:

$v^{0}(t+1)=v(t+1 \mid \hat{\theta}(t))=-\chi^{0}(t+1)=-x(t+1)-\hat{z}^{0}(t+1)$

where $\chi^{0}(t+1)$ is the measured residual acceleration. The "a posteriori" adaptation error (computed) will be given by:

$$
v(t+1)=v(t+1 \mid \hat{\theta}(t+1))=-x(t+1)-\hat{z}(t+1) .
$$

When using an estimated filter $\hat{N}$ with constant parameters: $\hat{y}^{0}(t)=\hat{y}(t), \hat{z}^{0}(t)=\hat{z}(t)$ and $v^{0}(t)=v(t)$.

The objective is to develop stable recursive algorithms for adaptation of the parameters of the $\mathrm{Q}$ filter such that the measured residual error (acceleration or force in AVC, noise in ANC) be minimized in the sense of a certain criterion. This has to be done for broadband disturbances $d(t)$ (or $s(t)$ ) with unknown and variable spectral characteristics and an unknown primary path model.

\section{Algorithm development}

The algorithm for adaptive feedforward compensation will be developed under the following hypotheses:

1. The signal $d(t)$ is bounded (which is equivalent to say that $s(t)$ is bounded and $W\left(q^{-1}\right)$ in figure 1 is asymptotically stable). 
2. It exists a central feedforward compensator $N_{0}\left(R_{0}, S_{0}\right)$ which stabilizes the inner positive feedback loop formed by $N_{0}$ and $M$ such that its characteristic polynomial ${ }^{3}$

$$
P_{0}\left(z^{-1}\right)=A_{M}\left(z^{-1}\right) S_{0}\left(z^{-1}\right)-B_{M}\left(z^{-1}\right) R_{0}\left(z^{-1}\right)
$$

is a Hurwitz polynomial.

3. (Perfect matching condition) It exists a value of the $Q$ parameters such that

$$
\frac{G \cdot A_{M}\left(R_{0}-A_{M} Q\right)}{A_{M} S_{0}-B_{M} R_{0}}=-D
$$

4. The effect of the measurement noise upon the measurement of the residual acceleration is neglected (deterministic context).

Once the algorithm will be developed under these hypotheses, hypotheses 3 and 4 are removed and the algorithm can be analyzed in this modified context.

A first step in the development of the algorithms is to establish for a fixed estimated compensator a relation between the error on the Q-parameters (with respect to the optimal values) and the adaptation error $v$. This is summarized in the following Lemma.

Lemma 1. Under the hypothesis 1, 2, 3 and 4 for the system described by eqs. (1) through (12), using a Q-parameterized feedforward compensator with constant parameters, one has:

$$
v(t+1 / \hat{\theta})=\frac{A_{M}\left(q^{-1}\right) G\left(q^{-1}\right)}{P_{0}\left(q^{-1}\right)}[\theta-\hat{\theta}]^{T} \phi(t),
$$

where $\theta, \hat{\theta}$ and $\phi$ are given respectively by:

$$
\begin{aligned}
\theta^{T} & =\left[q_{0}, q_{1}, q_{2}, \ldots, q_{n_{Q}}\right] \\
\hat{\theta}^{T} & =\left[\hat{q}_{0}, \hat{q}_{1}, \hat{q}_{2}, \ldots, \hat{q}_{n_{Q}}\right] \\
\phi^{T}(t) & =\left[\alpha(t+1), \alpha(t), \ldots, \alpha\left(t-n_{Q}+1\right)\right] . \\
\alpha(t+1) & =B_{M} \hat{y}(t+1)-A_{M} \hat{u}(t+1) \\
& =B_{M}^{*} \hat{y}(t)-A_{M} \hat{u}(t+1)
\end{aligned}
$$

$q_{i}$ are the coefficients of the optimal $Q$-filter and $\hat{q}_{i}$ are the coefficients of the fixed estimated $\hat{Q}$-filter.

For a proof, see Appendix A.

Filtering the vector $\phi$ by an asymptotically stable filter $L\left(q^{-1}\right)$, eq. (15) becomes

$$
v(t+1 / \hat{\theta})=\frac{A_{M}\left(q^{-1}\right) G\left(q^{-1}\right)}{P_{0}\left(q^{-1}\right) L\left(q^{-1}\right)}[\theta-\hat{\theta}]^{T} \phi_{f}(t)
$$

with

$$
\begin{aligned}
\phi_{f}(t) & =L\left(q^{-1}\right) \phi(t) \\
& =\left[\alpha_{f}(t+1), \alpha_{f}(t), \ldots, \alpha_{f}\left(t-n_{Q+1}\right)\right],
\end{aligned}
$$

\footnotetext{
${ }^{3}$ The parenthesis $\left(q^{-1}\right)$ will be omitted in some of the following equations to make them more compact.
}

\begin{tabular}{|c|c|c|}
\hline & $\begin{array}{c}\text { Present paper } \\
\text { (Fix IIR }+ \\
\text { Adaptive YKFIR) }\end{array}$ & $\begin{array}{c}\text { Landau et al. (2011a) } \\
\text { (Adaptive IIR) }\end{array}$ \\
\hline \hline$\hat{\theta}(t+1)=$ & $\hat{\theta}(t)+F(t) \psi(t) \frac{v^{0}(t+1)}{1+\psi^{T}(t) F(t) \psi(t)}$ \\
\hline $\begin{array}{c}\text { Adapt. } \\
\text { gain }\end{array}$ & $\begin{array}{c}F(t+1)^{-1}=\lambda_{1}(t) F(t)+\lambda_{2}(t) \psi(t) \psi^{T}(t) \\
0 \leq \lambda_{1}(t)<1,0 \leq \lambda_{2}(t)<2, F(0)>0\end{array}$ \\
\hline Adaptive & \multicolumn{2}{|c|}{ Decr. gain and const. trace } \\
\hline Self tuning & \multicolumn{2}{|c|}{$\lambda_{2}=$ const.,$\quad \lim _{t \rightarrow \infty} \lambda_{1}(t)=1$} \\
\hline$\hat{\theta}(t)=$ & {$\left[\hat{q}_{0}(t), \hat{q}_{1}(t), \ldots\right]$} & {$\left[-\hat{s}_{1}(t), \ldots, \hat{r}_{0}(t), \ldots\right]$} \\
\hline$\phi^{T}(t)=$ & {$[\alpha(t+1), \alpha(t), \ldots]$} & {$[-\hat{y}(t), \ldots, \hat{u}(t+1), \ldots]$} \\
\hline$\hat{P}=$ & $4(t)=B_{M} \hat{y}(t)-A_{M} \hat{u}(t)$ & $\hat{A}_{M} \hat{S}-\hat{B}_{M} \hat{R}$ \\
\hline$P=$ & $\hat{A}_{M} S_{0}-\hat{B}_{M} R_{0}$ & $A_{M} \hat{S}-B_{M} \hat{R}$ \\
\hline$A_{M} S_{0}-B_{M} R_{0}$ & $\hat{A}_{M} \hat{G}$ \\
\hline$\psi(t)=$ & \multicolumn{2}{|c|}{$L \phi(t) ; \quad L_{2}=\hat{G} ; L_{3}=\frac{\hat{A}}{\hat{P}}$} \\
\hline $\begin{array}{c}\text { Stability } \\
\text { condition }\end{array}$ & $\frac{A_{M} G}{P L}-\frac{\lambda}{2}=S P R$ & $\left(\lambda=\max \lambda_{2}(t)\right)$ \\
\hline $\begin{array}{c}\text { Conv. } \\
\text { condition }\end{array}$ & $\frac{A_{M} G}{P L}-\frac{\lambda}{2}=S P R \quad\left(\lambda=\lambda_{2}\right)$ \\
\hline
\end{tabular}

Table 1: Algorithms for adaptive feedforward compensation in AVC with mechanical coupling (YK parametrization and IIR parmetrization)

where

$$
\alpha_{f}(t+1)=L\left(q^{-1}\right) \alpha(t+1) .
$$

Eq. (17) will be used to develop the adaptation algorithms.

When the parameters of $\hat{Q}$ evolve over time and neglecting the non-commutativity of the time varying operators (which implies slow adaptation (Anderson et al., 1986) i.e., a limited value for the adaptation gain), equation (17) transforms into ${ }^{5}$

$$
v\left(t+1 / \hat{\theta}(t+1)=\frac{A_{M}\left(q^{-1}\right) G\left(q^{-1}\right)}{P_{0}\left(q^{-1}\right) L\left(q^{-1}\right)}[\theta-\hat{\theta}(t+1)]^{T} \phi_{f}(t) .\right.
$$

Eq. (20) has the standard form of an "a posteriori adaption error equation" (Landau et al., 2011b), which immediately suggests to use the following parameter adaptation algorithm:

$$
\begin{aligned}
\hat{\theta}(t+1) & =\hat{\theta}(t)+F(t) \psi(t) v(t+1) \\
v(t+1) & =\frac{v^{0}(t+1)}{1+\psi^{T}(t) F(t) \psi(t)} \\
F(t+1) & =\frac{1}{\lambda_{1}(t)}\left[F(t)-\frac{F(t) \psi(t) \psi^{T}(t) F(t)}{\frac{\lambda_{1}(t)}{\lambda_{2}(t)}+\psi^{T}(t) F(t) \psi(t)}\right] \\
1 & \geq \lambda_{1}(t)>0 ; 0 \leq \lambda_{2}(t)<2 ; F(0)=\alpha I ; \alpha_{\max }>\alpha>0
\end{aligned}
$$

$$
\psi(t)=\phi_{f}(t)
$$

where $\lambda_{1}(t)$ and $\lambda_{2}(t)$ allow to obtain various profiles for the adaptation gain $F(t)$ (see Landau et al. (2011b)).

\footnotetext{
${ }^{5}$ However, exact algorithms can be developed taking into account the noncommutativity of the time varying operators - see Landau et al. (2011b)
} 
Three choices for the filter $L$ will be considered:

$\begin{array}{ll}\text { Algorithm } I & L=G \\ \text { Algorithm } I I & L=\hat{G} \\ \text { Algorithm } I I I & \end{array}$

$$
L=\frac{\hat{A}_{M}}{\hat{P}_{0}} \hat{G}
$$

where

$$
\hat{P}_{0}=\hat{A}_{M} S_{0}-\hat{B}_{M} R_{0}
$$

A comparison with algorithms for IIR adaptive compensators (Landau et al., 2011a) is summarized in Table 1. For the IIR one adapts the filter parameters while for YK parametrized filters one adapts the parameters of the $\mathrm{Q}$ filter. For IIR, the regressor vector is constituted by filtered inputs and outputs while for YK parametrization, the components of the regressor vector are filtered linear combinations of input and outputs weighted by the parameters of the reverse path model.

\section{Analysis of the algorithms}

\subsection{The deterministic case - perfect matching} form:

Equation (20) for the a posteriori adaptation error has the

$$
v(t+1)=H\left(q^{-1}\right)[\theta-\hat{\theta}(t+1)]^{T} \psi(t),
$$

where

$$
H\left(q^{-1}\right)=\frac{A_{M}\left(q^{-1}\right) G\left(q^{-1}\right)}{P_{0}\left(q^{-1}\right) L\left(q^{-1}\right)}, \quad \psi=\phi_{f} .
$$

One has the following result:

Lemma 2. Assuming that eq. (24) represents the evolution of the a posteriori adaptation error and that the parameter adaptation algorithm (21a) through (21e) is used one has:

$$
\begin{gathered}
\lim _{t \rightarrow \infty} v(t+1)=0 \\
\lim _{t \rightarrow \infty} \frac{\left[v^{0}(t+1)^{2}\right]}{1+\psi(t)^{T} F(t) \psi(t)}=0 \\
\|\psi(t)\| \text { is bounded } \\
\lim _{t \rightarrow \infty} v^{0}(t+1)=0
\end{gathered}
$$

for any initial conditions $\hat{\boldsymbol{\theta}}(0), v(0)$ if:

$$
H^{\prime}\left(z^{-1}\right)=H\left(z^{-1}\right)-\frac{\lambda_{2}}{2}, \max _{t}\left[\lambda_{2}(t)\right] \leq \lambda_{2}<2
$$

is a strictly positive real (SPR) transfer function.

Proof: The proof is similar to that of (Landau et al., 2011a, Lemma 5.1) and is omitted.

The analysis in the presence of a measurement noise and when the perfect model matching does not hold can be carried on in a similar way as in Landau et al. (2011a) and it is omitted.

Remark 1: For algorithm III, the stability condition (30) for $\lambda_{2}=1$ can be transformed into (Ljung \& Söderström, 1983)

$$
\left|\left(\frac{A_{M}\left(e^{-j \omega}\right)}{\hat{A}_{M}\left(e^{-j \omega}\right)} \cdot \frac{\hat{P}_{0}\left(e^{-j \omega}\right)}{P_{0}\left(e^{-j \omega}\right)} \cdot \frac{G\left(e^{-j \omega}\right)}{\hat{G}\left(e^{-j \omega}\right)}\right)^{-1}-1\right|<1
$$

for all $\omega$, which is always true provided that the initial estimates of $M$ and $G$ are close to the true values (the differences between $P_{0}$ and $\hat{P}_{0}$ depend only upon the estimation errors of $\hat{M}$ ).

Remark 2: Consider eq. (15) for the case of time varying parameter $\hat{\theta}$. Neglecting the non-commutativity of time varying operators it can be written as:

$$
\begin{aligned}
v(t+1 \mid \hat{\theta}(t+1)) & =[\theta-\hat{\theta}(t+1)]^{T} \phi_{f}^{\prime}(t) \\
\phi_{f}^{\prime}(t) & =\frac{A_{M}\left(q^{-1}\right) G\left(q^{-1}\right)}{P_{0}\left(q^{-1}\right)} \phi(t)
\end{aligned}
$$

If one would like to minimize a one step ahead quadratic criterion $J(t+1)=v^{2}(t+1)$ using the gradient technique (Landau et al., 2011b) one gets

$$
\frac{1}{2} \frac{\partial J(t+1)}{\partial \hat{\theta}(t+1)}=-\phi_{f}^{\prime}(t) v(t+1)
$$

Using algorithm III, eq. (21a) can be viewed as an approximation of the gradient ( $F=\alpha I=$ const. for the gradient technique). For constant adaptation gain $\lambda_{2}(t) \equiv 0$ and the strict positive realness on $H^{\prime}\left(z^{-1}\right)$ implies at all the frequencies:

$$
-90^{0}<\angle \frac{A_{M}\left(e^{-j \omega}\right) G\left(e^{-j \omega}\right)}{P_{0}\left(e^{-j \omega}\right)}-\angle \frac{\hat{A}_{M}\left(e^{-j \omega}\right) \hat{G}\left(e^{-j \omega}\right)}{\hat{P}_{0}\left(e^{-j \omega}\right)}<90^{0}
$$

Therefore the interpretation of the SPR condition of Lemma 2 is that the angle between the direction of adaptation and the direction of the inverse of the true gradient should be less than $90^{\circ}$. For time-varying adaptation gains the condition is sharper since in this case $\operatorname{Re}\left\{H\left(e^{-j \omega}\right)\right\}$ should be larger than $\frac{\lambda_{2}}{2}$ at all frequencies.

Remark 3: The asymptotic bias distribution when perfect matching condition is not satisfied is given by (see Landau et al. (2011a) for the computation method):

$$
\hat{\theta}^{*}=\arg \min _{\hat{\theta}} \int_{-\pi}^{\pi}\left[\left|\frac{G(j \omega) A_{M}^{2}(j \omega)}{P_{0}(j \omega)}\right|^{2}|Q(j \omega)-\hat{Q}(j \omega)|^{2} \phi_{d}(\omega)+\phi_{w}(\omega)\right] d \omega
$$

where $\phi_{d}$ and $\phi_{w}$ are the spectral densities of $\mathrm{d}(\mathrm{t})$ and of the measurement noise. From (36) one concludes that a good approximation of $Q$ corresponding to the perfect matching will be obtained in the frequency region where $\phi_{d}$ is significant and where $G$ has a high gain (usually $G$ should have high gain in the frequency region where $\phi_{d}$ is significant in order to counteract the effect of $d(t)$ ). The quality of the estimated $\hat{Q}$ will be affected also by $A_{M}^{2} / P_{0}$.

Remark 4: In the case where some of the zeros of $\mathrm{G}$ are outside the unit circle, the use of Lemma 2 requires that the estimated unstable zeros be equal to the true unstable zeros and in addition that the minimal order transfer function $H^{\prime}$ be SPR. Extensive simulations have shown however that it is enough that real and estimated unstable zeros be sufficiently close in order that the phase condition associated to the positivity of the real part of $H^{\prime}$ is satisfied (even if $\mathrm{H}^{\prime}$ in this case can not be SPR).

\subsection{Comparison with IIR adaptive feedforward compensators}

Lets focus now on the differences between the IIR adaptive compensator given in Landau et al. (2011a) and the YK adaptive compensator. 
Remark 1: For IIR adaptive compensators, provided that the SPR condition is satisfied, the poles of the internal "positive" loop will be asymptotically stable but they can be very close to the unit circle (they can be inside of a circle of radius 0.99999. ..). This may induce some numerical problems in practice (when using truncation or fixed point arithmetic).

Remark 2: The central YK controller allows to assign the poles of the internal closed loop. Therefore one can impose that all the poles of the internal loop be inside of a circle of radius $1-\delta, \delta>0$ ( $\delta$ takes care of the numerical approximations $)$.

Remark 3: If a model based initial IIR compensator is available, it can not in general be used to initialize the parameters of the IIR adaptive compensator since often the number of parameters of the fixed compensator is higher than the number of parameters of the adaptive IIR compensator. The situation is different for YK adaptive compensator where any initial stabilizing compensator can be used whatever its complexity is.

Remark 4: For YK adaptive compensators the filters for Algorithm III can be directly implemented since the estimated closed loop poles are defined by the central controller and $\hat{M}$. For IIR adaptive compensators there is a need for an initialization horizon using Algorithm II followed by the real time computation of the estimated closed loop poles using $\hat{N}$ and $\hat{M}$.

\section{An active vibration control system using an inertial ac- tuator}

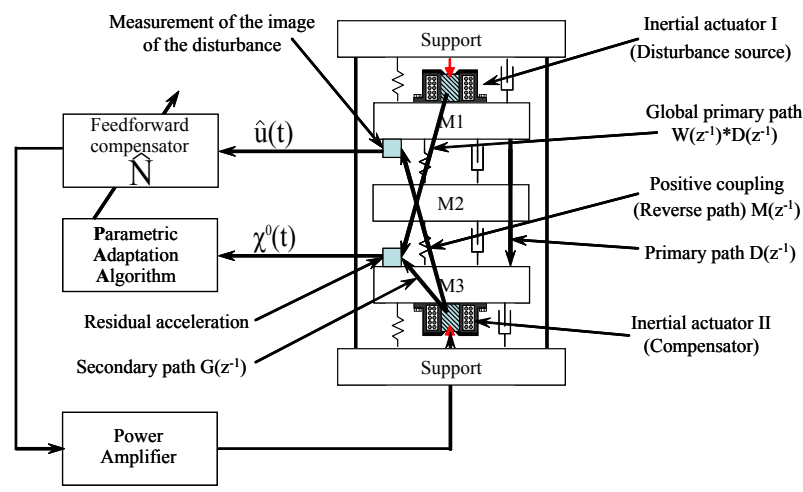

Figure 2: An AVC system using a feedforward compensation - scheme

Figure 2 represents an AVC system using a measurement of the image of the disturbance and an inertial actuator for reducing the residual acceleration which has been used for real time experiments. The system is composed of three metal plates interconnected by springs. The one on top (M1) is equipped with an inertial actuator which generates the disturbance $s(t)$ (figure 1). Another inertial actuator is located bellow plate M3 and is used for disturbance rejection. Two accelerometers positioned as in figure 2 measure the image of the disturbance and the residual acceleration $\chi^{0}(t)$. The corresponding block diagrams in open loop operation and with the compensator system are shown in figures 1(a) and 1(b). The procedure for identifying the various models has been described in Landau et al. (2011a). Their frequency characteristics are shown in figure 3. The model orders for the secondary path (solid line) and the reverse path (dotted line) have been estimated to be: $n_{B_{G}}=17, n_{A_{G}}=15$ and $n_{B_{M}}=16, n_{A_{M}}=16$ respectively. The primary path model has been used only for simulations.

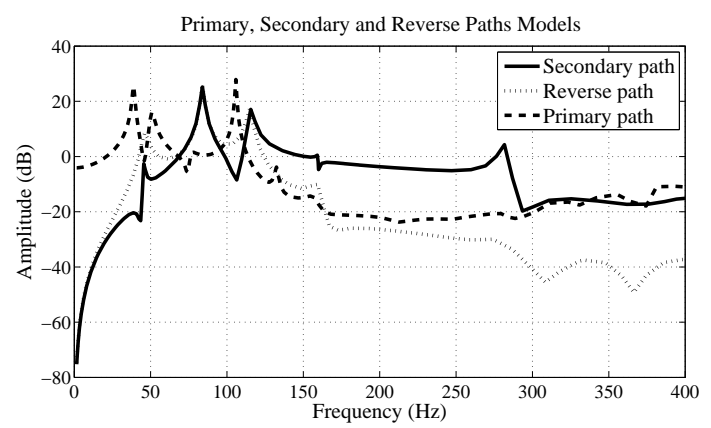

Figure 3: Frequency characteristics of the primary, secondary and reverse paths (identified models)

\section{Experimental results}

\subsection{The central controllers}

Two central controllers have been used to test this approach. The first (PP) has been designed using pole placement method. Its main objective is to stabilize the internal positive feedback loop. The end result was a controller of orders $n_{R_{0}}=15$ and $n_{S_{0}}=17$. The second controller is a reduced order $H_{\infty}$ controller with $n_{R_{0}}=19$ and $n_{S_{0}}=20$ from Alma et al. $(2011)^{6}$.

\subsection{Experimental results - Broadband disturbance rejection}

The broadband disturbance is a PRBS applied on the inertial actuator on top of the system. Its effect in the absence of the compensation system can be viewed in figures 4 and 5 (open loop power spectral density). Preliminary simulation studies

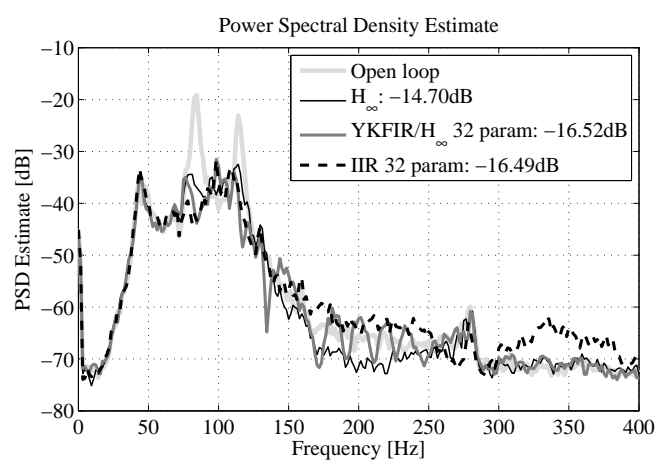

Figure 4: Experimental spectral densities of the residual acceleration $\left(H_{\infty}\right)$

have confirmed the theoretical expectations that algorithm III gives better results than algorithm $I I$. Subsequently only the algorithm III has been considered in the experiments. The power spectral densities obtained with the two central controllers without and with adaptation (32 parameters) are shown in figures 4

\footnotetext{
${ }^{6}$ The orders of the initial $H_{\infty}$ controller were: $n_{R_{H_{\infty}}}=70$ and $n_{S_{H_{\infty}}}=70$
} 


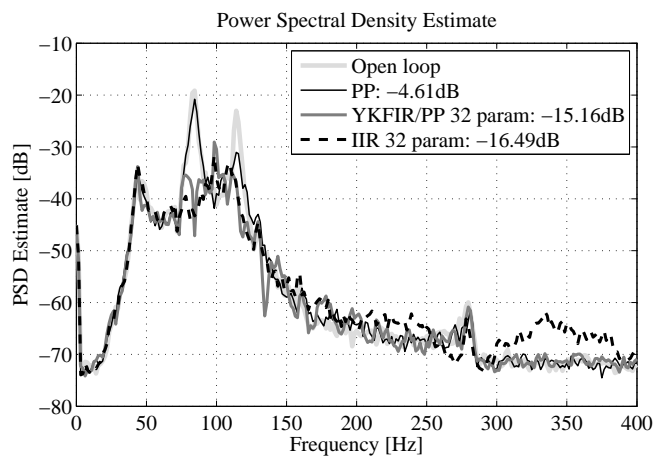

Figure 5: Experimental spectral densities of the residual acceleration (PP)

\begin{tabular}{|c|c|c|c|c|c|}
\hline No. of param. & 0 & 8 & 20 & 32 & 40 \\
\hline YK with $\mathrm{H}_{\infty}$ & $14.70 \mathrm{~dB}$ & $16.24 \mathrm{~dB}$ & $16.76 \mathrm{~dB}$ & $16.52 \mathrm{~dB}$ & $16.04 \mathrm{~dB}$ \\
\hline YK with PP & $4.61 \mathrm{~dB}$ & $14.26 \mathrm{~dB}$ & $14.49 \mathrm{~dB} 15.16 \mathrm{~dB} 15.56 \mathrm{~dB}$ \\
\hline IIR & - & $16.14 \mathrm{~dB}$ & $16.23 \mathrm{~dB}$ & $16.49 \mathrm{~dB}$ & $16.89 \mathrm{~dB}$ \\
\hline
\end{tabular}

Table 2: Influence of the number of parameters upon the global attenuation (experimental).

and 5. On both figures, the spectral density obtained using the IIR adaptive filter (Landau et al., 2011a), with 32 parameters, is also shown for comparison. Table 2 summarizes the global attenuation results obtained with the two central controllers for various number of parameters of the $\mathrm{Q}$ polynomial. The last line give the results for the IIR adaptive feedforward filter used in Landau et al. (2011a). In the column "0", the attenuations obtained for each structure, in the absence of the adapted filters, are given. For the YK parametrization, this corresponds to the use of the fixed central controller. For the IIR filter, this corresponds to open loop operation. For YK parametrized feedforward compensator the performance depends upon the central controller. For a well designed central controller, the performances are close to those of the IIR adaptive compensator.

\section{Conclusions}

FIR Youla Kucera parametrized adaptive feedforward compensators and IIR adaptive feedforward compensators provide close performances. However from a practical point of view the YK adaptive feedforward compensator seems more interesting in terms of initialization, assignment of the inner closed loop poles and implementation of the filters required by the positive real condition for stability and convergence.

\section{Appendix A. Proof of Lemma 1}

Under the assumption 3 (perfect matching condition) the output of the primary path can be expressed as

$$
x(t)=-G\left(q^{-1}\right) y(t),
$$

where $y(t)$ is a dummy variable given by

$$
y(t+1)=-S_{0}^{*} y(t)+R_{0} u(t+1)+Q\left[B_{M}^{*} y(t)-A_{M} u(t+1)\right]
$$

with

$$
u(t+1)=d(t+1)+\frac{B_{M}^{*}}{A_{M}} y(t) .
$$

The output of the adaptive feedforward filter (for a fixed $\hat{Q}$ ) is given by (7), where one replaces $\hat{Q}\left(t+1, q^{-1}\right)$ with $\hat{Q}\left(q^{-1}\right)$. The output of the secondary path is

$$
\hat{z}(t)=G\left(q^{-1}\right) \hat{y}(t) .
$$

Define the dummy error (for a fixed estimated set of parameters)

$$
\varepsilon(t)=y(t)-\hat{y}(t)
$$

and the residual adaptation error becomes:

$$
v(t)=-\chi(t)=-x(t)-\hat{z}(t)=G\left(q^{-1}\right) \varepsilon(t) .
$$

Equation (A.2) can be rewritten as

$$
\begin{aligned}
y(t+1)= & -S_{0}^{*} \hat{y}(t)+R_{0} \hat{u}(t+1)+Q\left[B_{M}^{*} \hat{y}(t)-A_{M} \hat{u}(t+1)\right] \\
& -S_{0}^{*}[y(t)-\hat{y}(t)]+R_{0}[u(t+1)-\hat{u}(t+1)] \\
& +Q\left[B_{M}^{*}(y(t)-\hat{y}(t))-A_{M}(u(t+1)-\hat{u}(t+1))\right] .
\end{aligned}
$$

Using (8) and (A.3) it results that

$$
Q\left[B_{M}^{*}(y(t)-\hat{y}(t))-A_{M}(u(t+1)-\hat{u}(t+1))\right]=0
$$

From equations (7) and (A.7) one obtains

$$
\varepsilon(t+1)=-S_{0}^{*} \varepsilon(t)+\frac{R_{0} B_{M}^{*}}{A_{M}} \varepsilon(t)+(Q-\hat{Q})\left[B_{M}^{*} \hat{y}(t)-A_{M} \hat{u}(t+1)\right] .
$$

Passing the terms in $\varepsilon(t)$ on the left hand side and taking into account eqs. (16d) and (A.6), one gets:

$$
v(t+1)=\frac{A_{M}\left(q^{-1}\right) G\left(q^{-1}\right)}{P_{0}\left(q^{-1}\right)}(Q-\hat{Q}) \alpha(t+1),
$$

Using eqs. (16a), (16b) and (16c), eq. (A.10) can be rewritten as eq. (15) which ends the proof.

\section{References}

Alma, M., Martinez, J. J., Landau, I. D., \& Buche, G. (2011). Design and tuning of reduced order h-infinity feedforward compensators for active vibration control. Control Systems Technology, IEEE Transactions on, PP, 1-8. DOI: 10.1109/TCST.2011.2119485.

Anderson, B., Bitmead, R., Johnson, C., Kokotovic, P., Kosut, R., Mareels, I., Praly, L., \& Riedle, B. (1986). Stability of adaptive systems. Cambridge Massachusetts, London, England: The M.I.T Press.

Elliott, S., \& Nelson, P. (1994). Active noise control. Noise / News International, (pp. 75-98).

Hu, J., \& Linn, J. (2000). Feedforward active noise controller design in ducts without independent noise source measurements. Control Systems Technology, IEEE Transactions on, 8, 443-455.

Jacobson, C., Johnson, C., Cormick, D. M., \& Sethares, W. (2001). Stability of active noise control algorithms. IEEE Signal Processing letters, 8, 74-76.

Kuo, M., \& Morgan, D. (1996). Active noise control systems-Algorithms and DSP implementation. New York,: Wiley.

Landau, I., Alma, M., \& Airimiţoaie, T. (2011a). Adaptive feedforward compensation algorithms for active vibration control with mechanical coupling. Automatica, 47, 2185 - 2196.

Landau, I. D., Lozano, R., M'Saad, M., \& Karimi, A. (2011b). Adaptive control. (2nd ed.). London: Springer.

Ljung, L., \& Söderström, T. (1983). Theory and practice of recursive identification. Cambridge Massachusetts, London, England: The M.I.T Press.

Zeng, J., \& de Callafon, R. (2006). Recursive filter estimation for feedforward noise cancellation with acoustic coupling. Journal of sound and vibration, 291, 1061-1079 